grateful to Dr Hughes for advice and instruction, and to the Corning Glass Works Foundation for a fellowship in support of the work.

\section{References}

Andress, K. R. \& GundermanN, J. (1934). Z. Kristal$\log r .87,345$.

Astashenko, K. L. \& Moleva, V. A. (1939). Miner. Abst. VII, 462.

Berghuts, J., Haanappel, IJ. M., Potters, M., LoopStra, B. O., MacGillavry, C. H. \& VeEnendaAL, A.L. (1955). Acta Cryst. 8, 478 .

Bernal, J. D. \& Megaw, H. D. (1935). Proc. Roy. Soc. $A, 151,384$.

BragG, W. L. (1937). Atomic Structure of Minerals, p. 140. Ithaca: Cornell Univ. Press.

Dana, E. W. (rev. Ford, W. E.) (1932). A Textbook of Mineralogy. New York: Wiley.

Dомониe, J. (1952). J. Phys. Chem. 56, 502.

Ewing, F. J. (1935). J. Chem. Phys. 3, 203.
HARKER, D. (1936). Z. Kristallogr. 93, 136.

Hoard, J. L. \& Grenko, J. D. (1934). Z. Kristallogr. $87,110$.

Kамв, W. B. (1960). Acta Cryst. 13, 24.

Megaw, H. D. (1934). Z. Kristallogr. 87, 185.

MeEs, C. E. K. (1954). The Theory of the Photographic Process, p. 166. New York: Macmillan.

Palache, C. (1932). Am. Min. 17, 304.

Pauling, L. (1933). Z. Kristallogr. 84, 442.

Pauling, L. (1939). The Nature of the Chemical Bond. Ithaca: Cornell Univ. Press.

Penfold, B. R. \& Grigor, J. A. Acta Cryst. In press. Schlaepher, M. \& Niggli, P. (1914). Z. anorg. Chem. $87,56$.

Smrth, J. V. (1954). Acta Cryst. 7, 479.

Takei, W. J. (1957). Thesis, Calif. Inst. of Technology. Wells, A. F. (1949). Acta Cryst. 2, 175.

Wells, A. F. (1950). Structural Inorganic Chemistry. Oxford: University Press.

Yoon, Y. K. \& Carpenter, G. B. (1959). Acta Cryst. 12, 17.

Acta Cryst. (1960). 13, 24

\title{
Accuracy of Atomic Positions in the Zunyite Structure
}

\author{
By W. Barclay Kamb \\ California Institute of Technology, Pasadena, California, U.S.A.*
}

(Received 14 February 1958)

\begin{abstract}
The accuracy of positional parameters in the refined zunyite structure is estimated by four different statistical methods, including a comparison of two entirely independent refinements of the structure. The estimates show tolerable agreement, but disagree as to the importance of $F_{o}$ measurement error in affecting the parameter error. Reliable estimates of $\pm 0.008 \AA$ (standard deviation) for oxygen coordinates and $\pm 0.003 \AA$ for silicon and aluminum coordinates are obtained.
\end{abstract}

\section{Introduction}

In a separate paper (Kamb, 1960) a detailed study of the structure of zunyite $\left(\mathrm{Al}_{13} \mathrm{Si}_{5} \mathrm{O}_{20}(\mathrm{OH})_{18} \mathrm{Cl}\right)$ is reported. Because of current interest in accurate interatomic distances in silicate structures, I considered it worthwhile to compare different statistical methods for estimating the accuracy of atomic positions in the refined zunyite structure.

There are four essentially independent ways in which the accuracy of atomic positions in the structure can be estimated: (1) a priori estimation of parameter variances by the method of Booth \& Britten (1948); (2a) a posteriori estimation from the agreement of observed and calculated intensities in the least-squares refinement, or $(2 b)$ from the final difference maps by the methods of Cruickshank $(1949 a, b) ;(3)$ comparison of the results of the independent $h k 0$ and $h h l$ refinements ; (4) comparison of independent $\mathrm{Si}-\mathrm{O}$ distances in

\footnotetext{
* Division of the Geological Sciences, Contribution No. 960
}

the same tetrahedron or in different tetrahedra if there is no preferential ordering of silicon and aluminum.

\section{A priori estimate}

The method given by Booth \& Britten (1948) and revised by Lipson \& Cochran (1953) enables a lower limit for the attainable parameter variances to be estimated from a knowledge of the measurement errors of the $F_{o}$ 's. Comparisons of two independent measurements of the $h k 0$ reflections, and also of the $h h l$, shows that for both sets of data the standard deviation estimate for the visually estimated logarithm of the intensity is 0.05 . For the average of two such independent measurements, the standard deviation estimate of the structure factors is $0.04\left|F_{o}\right|$. To use this information for an a priori prediction of the parameter variances by a relation of the type given by Lipson \& Cochran (1953), the high symmetry of the (100) projection used in the refinement must be 
taken into consideration. For an atom $j$ unrelated by symmetry to other atoms in the projection, the positional parameter standard deviation is estimated from

$$
\hat{\sigma}\left(x_{j}\right)=\hat{\sigma}\left(\frac{\partial \varrho}{\partial x}\right)_{j} /\left|\frac{\partial^{2} \varrho}{\partial x^{2}}\right|_{j}
$$

If only a center of symmetry is present, and if $\sigma\left(F_{o}\right)=$ $K\left|F_{o}\right|$, the standard deviation of the slope of the Fourier synthesis is simply

$$
\sigma\left(\frac{\partial \varrho}{\partial x}\right)=K\left\{\left\langle\left(\frac{\partial \varrho}{\partial x}\right)^{2}\right\rangle_{\Delta \nabla}\right\}^{\frac{1}{2}}=\sigma_{0},
$$

where the average is over the projection. For the (100) projection, of symmetry $p 4 m$, the Fourier synthesis is

with

$$
\varrho(x, y)=\frac{1}{A} \sum_{2} F_{h k 0} \cos 2 \pi h x \cos 2 \pi k y
$$

$$
F_{h k 0}=F_{\bar{h} k 0}=F_{\bar{h} \bar{k} 0}=F_{k h 0} .
$$

It is found that if $x \neq 0$ and $y \neq 0,(2)$ holds also for the density given by (3). In particular it holds for $x=y$, and hence gives directly the parameter standard deviations for $x_{1}$ ( $\mathrm{Si}_{\mathrm{II}}$ ) in zunyite (see Kamb, 1960), for $z_{5}\left(\mathrm{O}_{\mathrm{IV}}\right)$, and, neglecting the effect of overlap in the projection, for $x_{2}\left(\mathrm{O}_{\mathrm{I}}\right)$ and $x_{3}\left(\mathrm{O}_{\mathrm{II}}\right)$. The standard deviations for these parameters are of the type applicable to single resolved peaks in the asymmetric unit of the (100) projection, and will henceforth be called 'single peak standard deviations'. When $y=0$, then $\sigma(\partial \varrho / \partial x)=V(2) \sigma_{0}$, hence the parameter standard deviation for $\mathrm{O}_{\text {III }}$ is $/ 2$ times the single peak value. The standard deviations for atomic positions that, owing to the cubic symmetry, appear at more than one place in the asymmetric unit of the (100) projection can be estimated in the following way. The standard deviations for the separate peaks are estimated by the method used above, and a refinement process is then envisaged in which parameter shifts determined at the various peaks corresponding to a given point position are averaged in a way that minimizes the variance of the resulting averaged parameter shift. Factors derived in this way, for converting the actual expected standard deviations for different parameters in the structure to 'single peak' values, are listed in Table 1 under the designation $Q_{1}$.

If the projection contains $N$ peaks of Gaussian shape in elementary area $A$, so that

$$
\varrho(\mathbf{r})=\sum_{i}^{N} \varrho_{i}\left(\mathbf{r}-\mathbf{r}_{i}\right)=\sum_{i}^{N} \frac{Z_{i} p_{i}}{\pi} \exp \left[-p_{i}\left|\mathbf{r}-\mathbf{r}_{i}\right|^{2}\right]
$$

\begin{tabular}{|c|c|c|c|c|c|}
\hline \multirow{2}{*}{\multicolumn{2}{|c|}{ Parameter }} & \multirow{3}{*}{$\begin{array}{c}\text { Least } \\
\text { squares } \\
0.00066\end{array}$} & \multicolumn{2}{|c|}{ Conversion factors } & \multirow{2}{*}{$\begin{array}{l}\text { 'Single } \\
\text { peak' }\end{array}$} \\
\hline & & & $Q_{1}$ & $Q_{2}$ & \\
\hline $\mathrm{O}_{\mathrm{I}}$ & $x_{2}$ & & 1 & $0 \cdot 88$ & 0.00058 \\
\hline $\mathrm{O}_{\text {II }}$ & $x_{3}$ & 0.00077 & 1 & 0.78 & 0.00060 \\
\hline $\mathrm{O}_{\text {III }}$ & $z_{4}^{3}$ & 0.00085 & $1 / / 2$ & 1 & 0.00060 \\
\hline \multirow[t]{2}{*}{$\mathrm{O}_{\mathrm{IV}}$} & $x_{5}$ & 0.00059 & $\sqrt{2}$ & $0 \cdot 72$ & 0.00059 \\
\hline & $z_{6}$ & $0 \cdot 00060$ & 1 & 1 & 0.00060 \\
\hline \multirow[t]{2}{*}{$\mathrm{O}_{\mathrm{V}}$} & $x_{8}$ & 0.00033 & $\sqrt{3}$ & 1 & 0.00057 \\
\hline & $z_{6}$ & 0.0103 & - & - & - \\
\hline $\mathrm{Si}_{I I}$ & $x_{1}$ & 0.00022 & 1 & 1 & $0 \cdot 00022$ \\
\hline \multirow[t]{2}{*}{$\mathrm{Al}_{\mathrm{II}}$} & $x_{7}$ & 0.00017 & $ן^{\prime 2}$ & I & $0 \cdot 00024$ \\
\hline & $z_{7}$ & 0.00023 & I & 1 & 0.00023 \\
\hline
\end{tabular}

then (1) and (2) lead to

$$
\sigma\left(X_{j}\right)=K(\pi / 2)^{\frac{1}{2}} \frac{\left\{\frac{1}{A} \sum_{i}^{N} Z_{i}^{2} p_{i}^{2}\right\}^{\frac{1}{2}}}{Z_{j} p_{j}^{2}},
$$

Table 1. Estimated parameter standard deviations

where $\sigma\left(X_{j}\right)$ is given in the distance units used for $A$ and $p_{i}$. (5) differs from the corresponding relation of Lipson \& Cochran (1953), in the case that all the $p_{i}$ 's are the same, by a factor $2 / \sqrt{ } \pi$. The relation

$$
\frac{Z_{i} p_{i}}{\pi}=p_{i},
$$

where $\varrho_{i}$ is the height of the $i$ th peak, allows (5) to be written

$$
\sigma\left(X_{j}\right)=K(\pi / 2)^{\frac{1}{2}} \frac{\left(\frac{1}{A} \sum_{i}^{N} \varrho_{i}^{2}\right)^{\frac{1}{2}}}{p_{j} \varrho_{j}} .
$$

The application of $(6)$ to the (100) projection of zunyite leads to the predictions: for single oxygen peaks $\left(\mathrm{O}_{\mathrm{I}}\right.$ and $\mathrm{O}_{\mathrm{II}}$, neglecting effect of overlap), $\sigma(x)=0 \cdot 00032$; for single silicon or aluminum peaks (SiII), $\sigma(x)=0.00016$, the values being in units of the cell edge.

\section{A posteriori estimates}

Parameter standard deviation estimates from the $h k 0$ least-squares refinement (including off-diagonal matrix components) are given in Table 1 . The average standard deviation estimate for silicon and aluminum parameters is $0 \cdot 00021$, and for oxygen and hydroxide (except $z_{6}$, as explained below) $0 \cdot 00063$. The consistency of the values quoted can be judged by converting them to standard deviations corresponding to a single resolved peak in the asymmetric unit of the projection. The effect of overlapping of the peaks is removed by considering the contributions of the off-diagonal components of the normal equation matrix. The appropriate conversion factor for overlap for each parameter is listed under $Q_{2}$ in Table 1 . Standard deviations for point positions giving more than one peak in the asymmetric unit are converted to 'single peak' values by multiplying by the appropriate $Q_{1}$ factors from Table 1 . These factors can be derived directly from the structure factor and leastsquares formulae, and therefore do not depend on the particular Fourier refinement procedure envisaged in section 1 . The 'single peak' values (Table 4) cluster closely about the averages 0.00059 for $\mathrm{O}$ and $\mathrm{OH}$, and 0.00023 for Si and Al.

The least-squares estimate for the $z_{6}$ parameter 
standard deviation (Table 1) is abnormally high, due to the fact that $z_{6}$ is nearly 0 and therefore all derivatives $\partial F_{c} / \partial z_{6}$ are small. The $z_{6}$ standard deviation cannot be estimated adequately without going to a second order theory, but successive least-squares refinements show that $z_{6}$ is certainly less than 0.001 , and its standard deviation is probably no larger than that of other oxygen coordinates in the projection.

'Single peak' parameter variances estimated by the methods given by Cruickshank (1949 $a$, p. 72, equations 11.0 and 11.12) are given in Table 2. Curvatures $A_{h h}$ were calculated both by the method of Cruickshank $(1949 b$, p. 156, equation $3 \cdot 11)$ and directly from the Fourier synthesis with the help of the Gaussian approximation. The results of the two methods agree within about $15 \%$.

\section{Estimates from independent structural refinements}

Because the $h h l$ data were recorded, measured, reduced, and refined independently of the $h k 0$ data, a comparison of the results of the two refinements provides a check on the statistical estimates discussed above. Since in the $h h l$ refinement two different weighting systems were used, there are two final $h h l$ values for each parameter. Without distinguishing between these, and without distinguishing between significant and non-significant parameter shifts, the displacements from the $h k 0$ positions obtained in the $h h l$ refinements were combined to estimate 'single peak' standard deviations for the parameter shifts for oxygen atoms and (separately) for silicon and aluminum atoms. To estimate the 'single peak' standard deviations the shifts calculated in the refinements were converted to 'single peak' shifts by multiplying by appropriate factors $Q_{1}$ and $Q_{2}$ in Table 1 . The results are listed in Table 2.

Table 2. 'Single peak' parameter slandard deviations derived by various methods

\begin{tabular}{|c|c|c|c|}
\hline & Method & $\mathrm{O}, \mathrm{OH}$ & $\mathrm{Si}, \mathrm{Al}$ \\
\hline (1) & A priori & $0 \cdot 00032$ & 0.00016 \\
\hline$(2 a)$ & $A$ posteriori: Least squares & $0 \cdot 00059$ & $0.0002-3-3+3$ \\
\hline$(2 b)$ & Fourier & 0.00048 & 0.0002 \\
\hline & $\begin{array}{l}\text { Comparison of } h k 0 \text { and } h h l \\
\text { refinements }\end{array}$ & 0.00044 & 0.0001 \\
\hline
\end{tabular}

\section{Comparison of estimates}

Results of the four methods of estimating parameter accuracy, described above, are summarized in Table 2. The least-squares estimate agrees tolerably with the estimate from a comparison of the $h k 0$ and $h h l$ refinements. Although the $h h l$ projection is not centrosymmetric, the overall accuracy of coordinates derived from it is probably comparable to the accuracy of the $h k 0$ refinement, because the amount of data is more than twice as great, and because the resolution of separate atoms is more nearly complete. Moreover, there is no indication of a serious effect on the atomic positions due to the discrepancy between the temperature parameters for the $h k 0$ and $h h l$ data (see Kamb, 1960).

Comparison of the a priori and a posteriori estimates suggests that a large fraction $(0 \cdot 5-0 \cdot 7)$ of the error in the final coordinates is due to the errors of visual intensity estimation. This suggestion is supported by the Fourier standard deviation estimation procedure, but not by the least-squares procedure. In the Fourier procedure, the estimated standard deviation of the electron density gradient is $9 \cdot 1$ e. $\AA^{-3}$, while by the a priori procedure (method 1 ) it is $6 \cdot 1 \mathrm{e} . \AA^{-3}$. The least squares procedure provides a direct comparison of intensity measurement error with aggregate error from all sources. The estimated standard deviation of an $F_{o}$ with unit weight is

$$
\hat{\sigma}\left(F_{o}\right)=\left\{\Sigma w\left(F_{o}-F_{c}\right)^{2} /(u-v)\right\}^{\frac{1}{2}},
$$

where $u$ is the number of non-equivalent reflections and $v$ is the number of parameters adjusted in fitting $F_{c}$ to $F_{0}$. This estimate of the aggregate error is to be compared with the a priori estimate of the measurement error :

$$
\sigma_{M}\left(F_{o}\right)=K\left[\Sigma w F_{o}^{2}\right]^{\frac{1}{2}} .
$$

The $h k 0$ data give a least-squares estimate of 0.44 for the total error (in arbitrary units), while the a priori estimate of observational error is $0 \cdot 14$, suggesting that errors of visual intensity estimation contribute only about a third of the total error.

The discrepancy between two estimates of the importance of observational error cannot be satisfactorily accounted for. At first sight the discrepancy seems to be explained by the fact that the $a$ priori parameter standard deviation estimate is basically a Fourier estimate, and, as Cruickshank (1949b) has shown, Fourier estimates should always be larger than least-squares estimates of parameter accuracy, for the same standard deviation of the $F_{o}$ 's. But this explanation cannot be substantiated. An approximate calculation of $\sigma_{L} / \sigma_{F}$ (the ratio of the standard deviation of least-squares-refined and Fourier-refined coordinates) can be made from equation (5.3) of Cruickshank (1949b) if the multiplicity factors are omitted (they apply to only a small fraction of the total number of reflections), if the sums are replaced by integrals, and if the atomic $f$-values are taken as those due to Gaussian distributions of electron density (equation (4), above). With these approximations, one obtains for a complete set of 3-dimensional data for X-rays of wavelength $\lambda$

$$
\left(\sigma_{L} / \sigma_{F}\right)^{2}=15 \lambda^{5} p^{5 / 2} C_{3} / 32 \sqrt{ } 2 \pi^{9 / 2},
$$

and for a complete set of two-dimensional data

$$
\left(\sigma_{L} / \sigma_{F}\right)^{2}=\lambda^{4} p^{2} C_{2} / \pi^{4} .
$$


The $C$ 's are constants with values near to 1. For equation (7), which is of particular interest here,

$C_{2}=\left[1-\left(1+\alpha^{2}\right) \exp \left(-\alpha^{2}\right)\right]^{2} /\left[1-\left(1+2 \alpha^{2}\right) \exp \left(-2 \alpha^{2}\right)\right]$

where

$$
\alpha=2 \pi /\left(\lambda p^{\frac{1}{2}}\right)
$$

The result of (6), with $C_{3}$ assumed equal to 1 , $p=20 \AA^{-2}$ as found for most peaks in the zunyite projections, and $\lambda=0.71 \AA$ is $\sigma_{L} / \sigma_{F}=0.78$, which agrees with Cruickshank's (1949b) remark that improvement of about $25 \%$ in coordinate accuracy can be expected for weighted syntheses. Since the above data give $\lambda V p \cong \pi(7)$ results in

$$
\sigma_{L} / \sigma_{F} \cong V C_{2}=0.91
$$

Hence very little difference between least squares and Fourier estimates of coordinate accuracy is expected for the two-dimensional data of the zunyite study. In fact, the actual Fourier estimates (Table 2) are found to be slightly lower than the least-squares estimates, though probably not significantly lower.

The upshot of the above considerations is that some unknown internal inconsistency remains between the various methods of estimating parameter accuracy, and that it is not therefore possible to assess confidently the importance of intensity measurement error in the overall error of the refinement.

\section{Estimate from interatomic distances}

A comparison of the estimated standard deviations of interatomic distances can be made with the scatter of $\mathrm{Si}-\mathrm{O}$ distances in the $\mathrm{Si}_{5} \mathrm{O}_{16}$ group. There are three independent distances in the group:

$\begin{array}{llc} & \text { Dstimated } & \begin{array}{c}\text { Estandard } \\ \text { standation } \\ \text { deviation }\end{array} \\ \text { Atoms } & \text { Distance } & 0.018 \AA \\ \mathrm{Si}_{\mathrm{I}}-\mathrm{O}_{\mathrm{II}} & 1.64 \AA & 0.019 \\ \mathrm{Si}_{\text {II }}-\mathrm{O}_{\mathrm{II}} & 1.62_{5} & 0.007 \\ \mathrm{Si}_{\mathrm{II}}-\mathrm{O}_{\mathrm{V}} & 1.65 & \end{array}$

The scatter of the observed distances from a mean of $1.64 \AA$ corresponds to a standard deviation of $0.013 \AA$, which is to be compared with an average estimated standard deviation of $0.015 \AA$. Such close agreement is doubtless fortuitous.

\section{Accuracy. Summary}

The 'single peak' standard deviations of oxygen coordinates in zunyite can be taken confidently to be 0.00061 , corresponding to $\pm 0.0085 \AA$, and of silicon and aluminum 0.00023 , corresponding to $\pm 0.0032 \AA$. Bailey \& Taylor (1955), in the most accurate analysis of a complex silicate structure hitherto published, estimated from a three-dimensional Fourier refinement the standard deviations $\pm 0.0082 \AA$ for oxygen and $\pm 0.0031 \AA$ for silicon-aluminum. It is surprising that the standard deviations obtained in the two-dimensional refinement of the zunyite structure are so close to Bailey \& Taylor's estimates from three-dimensional refinement.

The agreement is due to the compensating effects of the greater number of reflections used in Bailey \& Taylor's analysis and the sharper atomic peaks ob. tained in the zunyite analysis. This can be verified in a rough but useful way by recognizing that the statistical uncertainties go as $N^{-\frac{1}{2}}$, where $N$ is the number of reflections used, whereas the effects of peak shape combine in such a way that for peaks containing the same number of electrons the standard deviation of a positional coordinate varies as $p^{-1}(p$ being the coefficient in the exponent of the Gaussian electron density) for two-dimensional refinement and as $p^{-5 / 4}$ for three-dimensional refinement, as shown in the a priori standard deviation estimates, so that for a rough comparison of the standard deviations for the two- and three-dimensional refinements we may take

$$
\frac{\sigma_{1}(x)}{\sigma_{2}(x)} \sim \sqrt{\frac{N_{2}}{N_{1}}}\left(\frac{p_{2}}{p_{1}}\right)^{9 / 8} .
$$

If in this expression we use the number of observed reflections $N_{1}=2652$ in Bailey \& Taylor's work and $N_{2}=133$ in the $h k 0$ refinement of zunyite, and take $p_{1}=5.4 \AA^{-2}$ (average of Bailey \& Taylor's values for $\mathrm{Si}$ and $\mathrm{O}$ ) and $p_{2}=20 \AA^{-2}$ as found for $\mathrm{Si}$ and $\mathrm{O}$ in zunyite, we obtain $\sigma_{1} / \sigma_{2}=1 \cdot 0$. It seems that the parameter accuracy obtainable with Bailey \& Taylor's data would be considerably (perhaps five times) greater if weighted least-squares refinement were used, making it unnecessary to introduce the high artificial temperature parameter $\left(B=4.0 \AA^{2}\right)$ that is responsible for the low curvature of the atomic peaks as compared to the peaks in zunyite.

\section{References}

Batley, S. W. \& Taylor, W. H. (1955). Acta Cryst. 8, 621.

Booth, A. D. \& Britten, K. H. V. (1948). Proc. Roy. Soc. A, 193, 305.

Cruickshank, D. W. J. (1949a). Acta Cryst. 2, 65.

Cruickshank, D. W. J. (1949b). Acta Cryst. 2, 154.

KAMB, W. B. (1960). Acta Cryst. 13, 15.

Lipson, H. \& Cochran, W. (1953). The Determination of Crystal Structures. London: Bell. 